\title{
A MODEL WHICH INTEGRATES STRATEGIC AND TACTICAL ASPECTS OF FOREST HARVESTING
}

\author{
Alastair McNaughton \\ Division of Science and Technology \\ Tamaki Campus, University of Auckland, \\ Private Bag 92019, New Zealand \\ a.mcnaughton@auckland.ac.nz
}

\author{
Mikael Rönnqvist \\ Division of Optimisation, \\ Linkoping University, \\ 58183 Linkoping, Sweden \\ miron@math.liu.se
}

\author{
David Ryan \\ Department of Engineering Science, \\ University of Auckland, \\ Private Bag 92019, Auckland, New Zealand \\ d.ryan@auckland.ac.nz
}

Abstract The forest harvesting problem involves the construction of a schedule for felling the individual blocks of trees which comprise a large commercial plantation. A strategic model sets long-term harvesting goals in terms of total area to be cut each year, but fails to identify individual blocks. A tactical model produces a short-term schedule of actual blocks. Until recently, most planning by forest managers has involved these as two separate models, often resulting in contradictory recommendations. We present an integrated model, which embraces both strategic and tacticial decisions, which can be solved by optimisation methods.

This model achieves a detailed formulation by means of a non-standard column generation structure. The solution algorithm solves the resulting relaxed linear program formulation. This is then combined

The original version of this chapter was revised: The copyright line was incorrect. This has been corrected. The Erratum to this chapter is available at DOI: 10.1007/978-0-387-35514-6_15

M.J.D. Powell and S. Scholtes (Eds.), System Modelling and Optimization: Methods, Theory and Applications. (C) 2000 IFIP International Federation for Information Processing.

Published by Kluwer Academic Publishers. All rights reserved. 
with constraint branching techniques to obtain the desired optimal integer solution to the integrated model.

Numerical output from a case study involving Whangapoua Forest in Coromandel, New Zealand, will be used to demonstrate the performance level of this algorithm in terms of the quality of optimisation, the size of the data base and the computational time. However, the main emphasis will be placed on the theoretical aspects of the model and its solution algorithm as the approach may well be transferable to other, quite distinct, applications.

\section{INTRODUCTION TO A CASE STUDY}

The Coromandel Peninsula is a magnificent bush-clad region of dissected hill country fringed by beautiful sandy coves and estuaries located in Northern New Zealand. Although the climate is generally warm with enough gentle rain to promote ideal growing conditions for trees, the location is prone to intense rainfall events and the associated risk of severe mass movement. Many tourists visit the Coromandel, making the preservation of the natural environment a top priority. The local community is deeply involved in conservation issues. The Whangapoua Forest occupies 7365 hectares on this peninsula. The forest is operated by Ernslaw One Ltd. The management of this company use a rotation of about 28 years. As a consequence, a horizon of about 30 years is needed for the long term strategic harvest planning. The strategic plan contains many constraints. Typical of these are the constraints resulting from the demanding environmental restrictions which limit the proportion of each catchment that may be harvested over any consecutive 5-year period. None of the strategic constraints concern specific blocks of trees. A second type of planning, called tactical planning, is needed for this purpose. Such detailed area-specific planning is required for a much shorter period of 2 to 5 years. At present the forest contains 145 surveyed mature blocks which contain trees representing about 30 crop types. This latter type of planning involves decisions on when each specific block will be felled. Each block must be felled as a single harvesting operation. It is not permitted to partially cut a block and then return later to complete the harvest. Further environmental and operational restrictions apply to this tactical planning. These include the so called cable logging adjacency requirements, which identify specific pairs or cliques of blocks and impose restrictions on when each member may be harvested in relation to the other members of the clique.

At present, harvest planning in the Whangapoua Forest is done in the following manner. Linear programming software is used to get an optimal strategic plan, followed by 2 or 3 days of manual activity to convert 
this into a feasible tactical plan for the next 2 or 3 years. The connection between the strategic plan and the tactical plan is very tenuous, so the optimal aspects of the strategic plan are lost. When the process is repeated 1 or 2 years later a totally different optimal strategic plan appears! Also forest managers are worried about issues of sustainability and long-term block-feasibility. For these reasons the Forest Research Institute was approached for advice, and our team, seeing this as an ideal research case study, became involved from early 1994.

\section{SALIENT CHARACTERISTICS}

The following distinctive characteristics of this application impact substantially on the model which follows. There are distinct short and long term planning tasks. A great many complex constraints are present in both situations. Mixed integer programming is required with large numbers of integer variables. Many of the management decisions are inter-related, such as road construction and removal of harvested logs. Terrain and the road network provide a partition of the forest into significant smaller units. There are problems associated with the sheer size of this application. The actual harvesting decisions concern a moderate number of blocks, 145, to be harvested over a small number of years, 6 . The excessive size of the application results from the combinatorial aspects of this situation. The model and the algorithm which follows may be transferable to other applications with similar characteristics.

\section{HISTORICAL SETTING}

Mathematical optimisation techniques have been applied to the solution of forest harvesting problems for over 30 years. During this time the nature of the problem has evolved. The growing power of the conservation movement has resulted in the inclusion of more and more constraints of greater severity and greater complexity. The physical size of planning tasks has greatly increased. The recent global drop in timber prices hess caused minimal profit margins and a compelling need to attain optimal, or near-optimal solutions if a competitive edge is to be maintained.

It is helpful to consider the literature in three main categories. First consider the optimisation stream. This includes basic papers applying linear programming to the strategic plan, such as that by Manley, Threadgill and Wakelin [9]. Realising that the tactical plan also had to be dealt with, attempts were made to extend a linear programme into a mixed integer programme. One of the best of these was that by Kirby, Hager and Wong [8], who developed the integrated resources planning model, (IRPM). This is a mixed integer formulation of an integrated 
forest harvesting model. The most singular achievement of IRPM is that it does find the optimal solution. Other researchers recognised the quality of Kirby's work but noted the limitations of IRPM to only small applications. O'Hara, Faaland and Bare [15] report IRPM can only deal with at most 50 integer variables along with a matrix of 250 rows and 250 columns.

One positive development has been to attempt to reduce the number of constraints by constraint aggregation. A number of papers such as those by Meneghin et al. [10], and Murray and Church [12] indicate that currently this is an approach widely regarded as promising. The present model includes constraint aggregation techniques in certain places.

When it became apparent that there were extreme difficulties in constructing an integrated model capable of the optimisation of an operational-sized application, a number of publications appeared advocating a hierarchical solution. These include Hof and Baltic [4], Weintraub and Cholaky [21], and Hof [5]. This approach involves first optimising the strategic plan, and then incorporating this strategic solution as a constraint in a separate optimisation of the tactical plan. Although the optimisation attained in this way will always be inferior to that obtained from an integrated model, the quality of the solution may be quite acceptable provided there are not too many constraints applicable to the tactical plan. Such an approach contains the assumption that, due to the aggregated nature of the variables used in the optimal strategic solution, a feasible solution will always be obtainable to the constrained tactical plan. In practice, this is almost never true, especially as the present trend continues for governments to impose ever more harsh area-sensitive environmental constraints on the tactical plan. As a consequence, the implementation of hierarchical solution algorithms has generally been accompanied by an observable deterioration in objective value between the strategic optimal solution and the final area-sensitive tactical solution. Daust and Nelson [1] have recorded this as a loss of between 4 percent and 20 percent. Such findings question the credibility of the optimisation process in these hierarchical models. An integrated model will avoid this problem.

The second category of forest harvesting research may be termed the heuristic stream. Here attention is focused on the integer variables, with many of these models relating exclusively to the tactical plan. A representative paper here would be that by O'Hara, Faaland and Bare [15]. They use a Monte Carlo method in which a subtle weighting is introduced to facilitate random generation of good quality solutions. The performance achieved is the heuristic solution, not necessarily optimal, 
of a problem containing 242 blocks, with a tactical horizon of 3 years, in 15 minutes on a VAX 8700 .

As one could well anticipate, this stream has indulged in ever more complicated heuristic methods. For example, a recent paper by Yoshimoto, Brodie and Sessions [24] proposes an intricate heuristic procedure in which the forest is partitioned into numerous sub-problems. Each subproblem is solved independently as a regional optimisation problem. A measure of the performance of this method is given by the solution of an application involving 10 time periods in 2 hours on a 486 PC.

Weintraub et al. [23] present a heuristic method they term heuristic integer planning, (HIP). This is based on the IRPM model of Kirby [8]. Kirby's optimisation problem has the disadvantage of producing output at the relaxed linear programme stage in which many of the integer variables have fractional values. HIP is a method that assigns some of these variables to value 0 or 1 . The problem is then re-optimised and more fractional variables are assigned integer values until an integer solution is obtained. The process is classified as heuristic because the assignment process is heuristic, being the enforcement of a set of rules. These rules may be either the outcome of the user's intuition or the trend observed from numerical trials. As the integer solution is obtained after 7 to 12 iterations it is clear that many variables must be assigned integer values every iteration. This model is capable of solving an application involving 44 road segments, and 28 blocks over a 3-period tactical horizon. A total of 190 integer variables is involved with the complete matrix containing 638 rows and 1071 columns. The solution process takes about 20 minutes.

A comparison such as that by Nelson and Brodie [13] of a heuristic with an optimisation method is very insightful. In this paper a forest harvesting application involving 45 blocks, 52 road segments and 3 tirne periods, that is 291 integer variables, is first solved to optimality by a mixed integer programme algorithm. Using two computers, a 80386PC and a hyper-LINDO PC this is achieved in 60 hours. The same application is then solved by a Monte-Carlo integer programming (MCIP) heuristic in 9 hours, but the best objective value obtained is 97 percent of the optimal value obtained from the optimisation method.

Sessions and Sessions [17] have developed heuristic software that produces a tactical plan without any reference to the strategic plan. The tactical model is formulated and solved first without including adjacency constraints. Monte Carlo methods are used to generate large numbers of candidate solutions that are then checked against adjacency constraints to determine which, if any, are feasible. The resulting software is named SNAP II. John Sessions has collaborated with Nelson and Brodie [14] 
to investigate techniques to use SNAP II in association with the linear programming strategic software FORPLAN. Since these two packages are quite distinct an integrated optimisation is extremely difficult.

The third and final division consists of multiple-stage methods. These also deal primarily with the tactical plan and the difficulties relating to the integer variables contained in it. A number of recent papers recommend methods that consist of several stages, with different methods at each stage. One of the best of these would be that by Weintraub, Barahona and Epstein, [22] who suggest a column-generating technique to solve a forest harvesting model. Unfortunately, even after some major simplification assumptions, the formulation leads to a series of very difficult and complex sub-problems for which an optimal solution cannot be attained. Probably the most serious weakness of this type of approach is the extreme difficulty of driving a multi-stage method close to optimality. The task of trying to establish a sound theoretical foundation for such a structure is daunting. Weintraub et al report successful implementation of their algorithm on a number of operational Chilean forests, but unfortunately do not include the computing times involved.

Another significant multiple-stage model is that of Hoganson and Borges [7], who apply dynamic programming techniques to a multitude of subproblems. Other recent formulations include those of Snyder and ReVelle $[19,20]$. These contain interesting mathematical insights, but have the weakness that they are presented in the context of hypothetical integerised grid data. Hof, Bevers and Pickens [6] utilise a similar digitised grid, but they show how this is derived from a real map of an actual forest. Naturally their grid cells do not correspond to actual cutting blocks, a factor that must cause significant difficulties when any adjacency constraints are needed. Hochbaum and Pathria [3] also use a grid structure for their model. It is noteworthy that they attempt to negotiate these difficulties involving adjacency constraints, by penalising, rather than prohibiting, infringements of adjacency restrictions.

Sherali, Adams and Driscoll [18] present a helpful generalised theoretical model which stresses the importance of attaining a tight linear programming relaxation, which curiously is a feature of the model to be presented below.

The most salient aspect of this substantial body of research literature is its extreme diversity. This indicates that despite much excellence and insight, not one of the many and varied solution methods has won any degree of wide-spread acceptance. This is a strong indicator that the forest harvesting problem is at present unsolved in an optimisation sense. 
One extremely important related problem is that of scheduling the flights of airline crews into tours of duty, along with the related problem of rostering crews to these tours of duty. In simple terms, the models involved are huge mixed integer structures. Each possible roster for a crew member is represented by a column. Binary variables are used to determine which crew member is assigned to which roster. When compared with forest harvesting problems there are fundamental differences. The crew scheduling problem belongs to the class known as set-partitioning problems, whereas forest harvesting problems do not. However, the important factor is that recent major advances have occurred in the technology of solving set-partitioning problems. These involve column generation in association with constraint branching. Desrochers et al [2] and Ryan [16] have developed these methods so as to obtain optimisation solutions to applications previously thought too large for anything other than a heuristic treatment. As a consequence, very definite and significant practical improvements are now available to airline management. The solution algorithm of McNaughton [11] applied constraint branching to the forest harvesting problem for the first time.

\section{MAIN FEATURES OF THE PROPOSED MODEL}

An integrated model has been chosen so as to get maximum benefits from optimisation, and to avoid conflict between tactical and strategic plans. This means that the strategic part of the plan, which involves continuous variables, will be joined by linking constraints to the tactical part of the plan which contains binary integer variables. An objective function will be used which represents the present net worth of the forest, with revenue and costs from planned future harvests discounted appropriately. It is significant that the revenue part of the objective is associated with the strategic part of the model, while the costs are mostly generated by the tactical part. The solution will then be obtained by a single optimisation process acting on the whole model. We will allow 30 years for the strategic plan and 6 years for the tactical plan, using a time period of 1 year. The strategic part of the model will contain about 1820 continuous variables and about 1120 constraints. The tactical part of the model will contain about 1560 binary integer variables and about 1100 constraints. Although it is not possible to list every one of these constraints in detail, the most significant of them will be displayed and explained in Section 5. Road decision variables will be included in the tactical plan. Many more binary integer variables will be introduced 
by column generation during the solution process as will be explained below.

\section{SOME MODEL DETAIL}

The variables in the strategic part of the model, $x_{c e t}$, are continuous, with

$x_{c e t}=$ hectares of croptype $c$ established in year $e$ harvested in year $t$.

These are used to construct constraints in the strategic plan, which has the structure of a linear programme. For example, there may be a requirement that no more than 200 hectares be felled in a year. This would involve the maximum area constraint

$$
\sum_{c e} x_{c e t} \leq 200 \text {. }
$$

This constraint, as with the others which follow, is representative of a large block of constraints. In this case a separate constraint is required for each establishment year for each croptype in the forest. The other constraints in the strategic part of the model are mostly of equally simple formulation. These concern matters such as acheiving a non-declining yield and ensuring that no more of a given croptype is harvested than is currently available. Since the structure of this part is that of a linear programme, it poses no significant difficulties in the solution process, other than those associated with its size, comprising as it does of many hundreds of constraints.

For the tactical part of the model the formulation is much more complex. The following key concept is of pivotal importance in the model as it provides the definitions for the principle decision variables. A road harvest plan is a set of tactical decisions all pertaining to the harvesting of blocks on one given road. These may span the entire tactical horizon. Only one road harvest plan will be permitted to be operating in the solution for each road, although many alternative road harvest plans may be generated in the model. Here is an example of a road harvest plan:

On road 14, cut block 2 in year 3 , block 3 in year 2 , and block 5 in year 6 .

In the linear programming matrix representation of the model, a unique column with an associated integer variable will be associated with each road harvest plan. In this case the variable $g_{j t n}$ is used.

$$
g_{j t n}=\left\{\begin{aligned}
1 & \begin{array}{r}
\text { if the } n \text {-th plan on road } j \text { is chosen, } \\
\text { harvesting starting in year } t,
\end{array} \\
0 & \text { if the } n \text {-th plan on road } j \text { is not chosen. }
\end{aligned}\right.
$$


For each road, one of these variables will represent a null harvest plan in which no harvesting occurs during the planning horizon.

Other columns, also with associated integer variables, represent road construction tasks. In this case the variable $r_{j t}$ is used.

$r_{j t}= \begin{cases}1 & \text { if road } j \text { is constructed in year } t \\ 0 & \text { if road } j \text { is not constructed in year } t .\end{cases}$

The structure of the integrated model ensures that an optimal integer solution to the associated road problem is automatically produced without any explicit manipulation of these parts of the matrix, provided an optimal integer solution is obtained for the road harvest plan variables.

Two of the tactical constraints have a pivotal role in the solution algorithm and need to be studied closely. Firstly a plan constraint is required for each road. This will ensure one and only one road harvest plan is selected for a particular road, $j_{o}$, say. These constraints are of the form:

$$
\sum_{t} \sum_{n} g_{j_{o} t n}=1
$$

Secondly, road construction constraints are used to allow harvesting on road $j_{o}$ only after this road has been constructed. If $n^{*}$ represents the null harvest plan, then these are of the form:

$$
-\sum_{\bar{t}=1}^{t} r_{j_{o} \bar{t}}+\sum_{n \neq n^{*}} g_{j_{o} t n} \leq 0 .
$$

Other types of tactical constraints are of interest too. Road sequential constraints are required to ensure that a continuous access route is available from the harvesting site to the timber mill. Suppose either road $j_{1}$ or $j_{2}$ is needed to provide access to road $j_{3}$. Then the necessary constraints will be of the form

$$
-\sum_{\bar{t}=1}^{t}\left(r_{j_{1} \bar{t}}+r_{j_{2} \bar{t}}\right)+r_{j_{3} t} \leq 0 .
$$

The construction of the linking constraints is of major significance. Recall that the model consists of two approximately equal parts, one generating revenue, the other cost. They involve quite different variables and are completely disjoint apart from the linking constraints. If these are used to impose a strict equality between the croptype areas, designated by the continuous variables, $x_{c e t}$, in the strategic part, and the areas of the appropriate specific blocks chosen for harvest, represented 
by expressions involving the integer variables, $g_{j t n}$, in the tactical part, then the model becomes too stiff. In this case the performance of the solution algorithm is greatly impaired. On the other hand, if slack variables are introduced the solution algorithm runs fast but the integrity of the optimisation is violated. Instead new continuous variables, $s_{c e t}$, called overlap variables, are introduced.

$$
\begin{aligned}
s_{c e t}= & \text { the area of croptype } c \text { established in year } e \text { harvested } \\
& \text { in year } t+1 \text { which is located in blocks where harvesting } \\
& \text { will commence in year } t .
\end{aligned}
$$

Let the constant $a_{c e j k}$ represent the area of croptype $c$ established in year $e$ located in block $k$ on road $j$. Let $G_{j t k}$ be the set of all $g_{j t n}$ which involve the harvesting of block $k$ on road $j$ in year $t$. The linking constraints will then be of the form

$$
-x_{c e t}-s_{c e t}+s_{c e[t-1]}+\sum_{j k} a_{c e j k} \sum_{G_{j t k}} g_{j \bar{t} n}=0 .
$$

The optimisation process, in association with the discounted nature of the objective and the requirement that the tactical variables be integer, will ensure that only a very few of these overlap variables, $s_{c e t}$, are nonzero in the final solution.

Finally there will be many adjacency constraints which record restrictions on the permitted harvesting time for a block in relation to that of a given neighbouring block. As an example suppose that for technical reasons the logging of block $a$ on road $j_{a}$ required the logging of block $b$ on road $j_{b}$ within at most 2 years. This occurs if a harvesting technique called cable logging is being used and certain local terrain conditions apply. Let the tactical horizon be 3 years. Then the necessary adjacency constraint would be

$$
\begin{aligned}
-1 \leq 3 \sum_{G_{j_{a} 1 a}} g_{j t n}-3 \sum_{G_{j_{b} 1 b}} g_{j t n}+2 \sum_{G_{j_{a} 2 a}} g_{j t n} \\
-2 \sum_{G_{j_{b} 2 b}} g_{j t n}+\sum_{G_{j_{a} 3 a}} g_{j t n}-\sum_{G_{j_{b} 3 b}} g_{j t n} \leq 1 .
\end{aligned}
$$

\section{SOLUTION ALGORITHM}

During the solution algorithm the difficulties associated with the integer variables are circumvented by considering the relaxed linear programme in which each binary variable is considered as a continuous variable, bounded to the interval $[0,1]$. This allows extensive use of standard 
linear programming techniques throughout the solution process. In addition two other processes are involved.

The first of these is column generation. If every possible road harvest plan was to be represented explicitly by a column in the matrix representation of the linear programme, then the size of the matrix would be too large. So at first only a few representative columns are included. In the present model these correspond to elementary road harvest plans, $e_{j t k}$, which each concern the harvesting of just one single block in a given year.

$$
e_{j t k}= \begin{cases}1 & \text { if block } k \text { on road } j \text { is cut in year } t \\ 0 & \text { otherwise }\end{cases}
$$

The variables $e_{j t k}$ are a subset of the more general $g_{j t n}$. Each iteration new columns are added to the linear programme matrix, each representing a road harvest plan. Each new column will be a composition of 2 or more of these elementary road harvest plans, which is selected to improve the current objective value, until an optimal relaxed linear programme solution is obtained. In this model a non-standard type of column generation has been used which delivers a high performance level. The role of the $e_{j t k}$ variables in this column generation will be explained in the next section.

The second process is constraint branching. This is a special type of branch and bound. It is used to remove fractional values from the solution to the relaxed linear programme. It works much faster than traditional variable branching, but can only be used if the model has been constructed appropriately.

During the implementation of the solution algorithm these two processes work together. First a phase of column generation is used to solve the relaxed linear programme. Then a suitable constraint branch is implemented. The problem is then re-optimised with a further phase of column generation as the new constraint branch may well have made certain new columns desirable. The rapidity of the column generation process makes this easy. If the new relaxed linear programme has an integer solution then we stop. Otherwise another constraint branch is chosen and the process continues iteratively.

\section{TECHNICAL DETAILS OF THE COLUMN GENERATION}

When a linear programme is being solved by the revised simplex method, a vector called the reduced cost is obtained. The reduced cost of a basic variable is always 0 . So it is only necessary to define the reduced cost for the non-basic variables. Let the linear programme be of 
the form

$$
\text { minimise } c^{T} x \text { subject to } A x \leq b, 0 \leq x
$$

Let $B$ be the submatrix of basic columns of $A, c_{\mathrm{B}}$ be a vector of objective coefficients of the basic variables, $A_{\mathrm{N}}$ be the submatrix of non-basic columns of $A$, and $c_{\mathrm{N}}$ be a vector of objective coefficients of the nonbasic variables. The reduced cost vector, $r c$, is defined by

$$
r c=c_{\mathrm{N}}^{T}-c_{\mathrm{B}}^{T} B^{-1} A_{\mathrm{N}}
$$

In the revised simplex method a negative component of the reduced cost vector indicates that the corresponding variable may be added to the basis without causing a deterioration of the objective value. A reduced cost vector which is entirely non-negative is taken as the test for optimality.

When a column generation method is being used, the matrix $A$ contains only a representative sample of all the many possible columns the application permits. In this case the reduced cost may be evaluated for any of the other columns which are being considered as possible entering columns. Once again, a negative reduced cost indicates that this new column may be added to matrix $A$, in fact to the basis in $A$, without causing a deterioration of the objective value. If it can be shown that no entering column with a negative reduced cost exists, then this may be used as a test for optimality. Unfortunately, the definition in Equation 7 is unsuitable for use in a column generation context, as the set of all possible entering columns is not explicitly accessible. Also the task of evaluating the reduced cost for every such column would generally be unacceptably tedious.

Most column generation methods deal with this problem by constructing a subproblem designed to produce an entering column with a negative reduced cost. These subproblems are of varied types, depending on the nature of the application, but are generally complicated and difficult to solve. A good example of this has already been noted in the work of Weintraub et al [22]. In the present model, information obtained from the solution to the current linear programme is used to determine the minimum reduced cost of any entering column. The solution to a linear programme includes both a value of the reduced cost variable, $r c$, associated with each variable, and also a value of the dual variable, $\pi$, associated with each constraint. A vector of dual variable values may be defined by

$$
\pi=c_{\mathrm{B}}^{T} B^{-1}
$$


The dual variables and the reduced cost variables are related by the following equation, where $A=\left(a_{i j}\right)$, a matrix with $n$ rows.

$$
r c_{j}=c_{j}-\sum_{i=1}^{n} a_{i j} \pi_{i}
$$

Equation 9 allows the reduced cost of any entering column to be determined. Unfortunately it requires the new column to be constructed explicitly before the reduced cost can be computed. This would be unsatisfactory in view of the very large number of possible entering columns. So a refinement of Equation 9 will be derived which will allow a reduced cost to be found without having to construct the new column.

Recall that the initial $A$ matrix contains one column for each elementary road harvest plan. Each entering column will be a composite road harvest plan involving the harvesting of 2 or more blocks on the same road. When such a composite column is formed, most entries will be a simple vector addition of the corresponding entries in the appropriate elementary columns. If this were true for all entries, then the reduced cost of the entering column would be merely the sum of the known reduced costs of these elementary columns. However, in the case of the plan constraints, and any trigger constraints present such as the road construction constraints, the composite column is not formed as a sum, but rather as the maximum of the set of corresponding entries.

Consider the column corresponding to a road harvest plan $g_{j t n}$. From Equation 2 this column will contain an entry of 1 in the plan constraint associated with road $j$. Let the associated dual variable be $\pi_{j}$. Similarly, from Equation 3 this same column will contain an entry of 1 in each of the road construction constraints associated with road $j$ from year $t$ to $t^{\prime}$, where $t^{\prime}$ is the end of the tactical planning horizon. Let the dual variables associated with these road constraints be $\pi_{j t}$.

Now consider $\overline{r c}\left(g_{j t n}\right)$, the reduced cost of a column, corresponding to a road harvest plan $g_{j t n}$, in which the entries for the plan constraints and the road construction constraints are removed. Since this truncated column is the vector sum of the appropriate truncated elementary columns, $\overline{r c}\left(g_{j t n}\right)$ is the sum of the reduced costs of these truncated elementary columns.

$$
\overline{r c}\left(g_{j t n}\right)=\sum_{e_{j t k} \sim g_{j t n}} \overline{r c}\left(e_{j t k}\right)
$$


Also, it follows from Equation 9 that the truncated reduced cost for an elementary column, $\overline{r c}\left(e_{j t k}\right)$, is readily computed by

$$
\overline{r c}\left(e_{j t k}\right)=r c\left(e_{j t k}\right)+\pi_{j}+\sum_{\bar{t}=t}^{t^{\prime}} \pi_{j \bar{t}} .
$$

Let $g_{j t n}$ represent a composite plan with block $k_{i}$ harvested in year $t_{i}$. Observe $t=\min \left\{t_{i}\right\}$. Then

$$
\begin{array}{rlr}
r c\left(g_{j t n}\right) & =\overline{r c}\left(g_{j t n}\right)-\pi_{j}-\sum_{\bar{t}=t}^{t^{\prime}} \pi_{j \bar{t}} & \\
& =\sum_{e_{j t_{i} k} \sim g_{j t n}} \overline{r c}\left(e_{j t_{i} k}\right)-\pi_{j}-\sum_{\bar{t}=t}^{t^{\prime}} \pi_{j \bar{t}} & (\text { as in (11)) } \\
& =\sum_{e_{j t_{i} k} \sim g_{j t n}}\left(r c\left(e_{j t_{i} k}\right)+\pi_{j}+\sum_{\bar{t}=t_{i}}^{t^{\prime}} \pi_{j \bar{t}}\right)-\pi_{j}-\sum_{\bar{t}=t}^{t^{\prime}} \pi_{j \bar{t}}
\end{array}
$$

Equation 12 allows the minimum reduced cost for any entering column to be determined merely by scanning the known values of the reduced costs of the elementary columns and the dual variables of the appropriate trigger constraints. Any elementary column excluded by the active constraint branches is omitted from this scan. If this minimum value is negative, then the appropriate new column can be easily constructed and added to the matrix. If this minimum value is non-negative, optimality has been attained.

\section{TECHNICAL DETAILS OF THE CONSTRAINT BRANCHING}

Although $r_{j t}$ and $g_{j t n}$ are all binary integer variables, during the solution process the problem is treated as just one large relaxed linear programme. This is necessary whenever column generation techniques are to be applied. Integer solutions are obtained by a branch and bound process. Until recently, this was generally done by a process called variable branching in which a single binary variable was adjusted to a value of 1 , on the 1 -branch, or 0 , on the 0 -branch. The use of variable branching results in a slow convergence to an integer solution with a massive binary tree requiring to be searched.

Instead of variable branching, the present algorithm uses constraint branching, similar to that developed by Desrochers et al [2] and Ryan 
[16]. In the forest harvesting application this entails using the sets $G_{j t k}$ which have already been introduced in relation to the linking constraints of Equation 5 and the adjacency constraints of Equation 6. For example, a typical branching node consists of a 0 -branch

$$
\sum_{t \leq T}\left[\sum_{G_{j t k}} g_{j \overline{t n}}\right]=0
$$

and a 1-branch

$$
\sum_{t \leq T}\left[\sum_{G_{j t k}} g_{j \bar{n} n}\right]=1 .
$$

Thus the 0-branch prevents the adoption of any road harvest plan in which block $k$ is felled by year $T$. The 1-branch requires that one of these harvest plans must be chosen, without specifying exactly which of all the many eligible plans this will be. One compelling reason for the use of constraint branching is the dramatic improvement in computational time which results. However, this technique cannot be applied unless the model has been formulated in an appropriate manner. In the present case the necessary sets of road harvest plans, $G_{j t k}$, have been carefully built into the model for this purpose.

A most helpful enhancement of constraint branching concerns what may be called allied constraint branches. These involve the identification and automatic implimentation of any other constraints that are in sorne way logical consequences of the chosen constraint branch. For example, in forest harvesting an operational requirement may force a pair of adjacent blocks to be felled within two years of each other, as in Equation 6 . Let the blocks be $a$ and $b$ on roads $j_{a}$, and $j_{b}$ respectively. In this case if the branch

$$
\sum_{t \leq T}\left[\sum_{G_{j_{a} t a}} g_{j_{a} \bar{t} n}\right]=1
$$

is applied, then so too should be the allied branch

$$
\sum_{t \leq T+1}\left[\sum_{G_{j_{b} t b}} g_{j_{b} \bar{t} n}\right]=1 .
$$

Effort spent searching out allied constraint branches is amply rewarded by very significant reductions in computational time.

Another good feature of this constraint branching procedure is that it involves the use of only one type of decision node. It is not necessary to 
impose separate branches using the road variables, as the model has been constructed in such a way that once all the sets of variables designated by $G_{j t k}$ have been forced to integer sums then all other non-continuous components will automatically assume integer values. As a result of this the preferred direction in the branch and bound process is readily apparent, allowing a good quality integer solution to be attained at a relatively shallow depth.

Applications which involve column generation almost always require the use of branch and bound to obtain an integer solution. Consequently, the evaluation of a column generation technique should take into account the associated branch and bound process. In the forest harvesting application, the value of the column generation would be largely wasted without the constraint branching. During the implementation of the solution algorithm both these processes work together with alternating phases of column generation and constraint branching as described in Sections 6 and 7 . The quality of the resulting solution will next be presented.

\section{PERFORMANCE LEVELS ATTAINED IN CASE STUDY}

The main output from this case study includes 5 complete years of a detailed cutting plan, a road construction schedule to match, and a harmonised 30 year strategic plan. An optimal integer solution is obtained at a depth of about 80 nodes, after about 6 minutes computational time on a solaris-2.6 (sparc) computer, with an objective value of 99.95 percent that of the relaxed linear programme.

The robustness of the model has been tested by numerical trials in which various parameters of the problem have been increased. For example, the number of years in the tactical plan has been increased from 3 to 10 . Also the number and type of adjacency constraints has been varied. From 0 to 132 cable logging adjacency constraints of the form given in Equation 6 have been imposed. Also, up to 144 alternative green-up adjacency constraints have been used. These prevent the harvesting of adjacent blocks within a green-up period of 5 years. Such modifications result in changes to the objective value, but in each case the optimal objective value remains within half of one percent of that of the corresponding relaxed linear programme. This indicates that this is a tight linear programme relaxation, as advocated by Sherali et al [18].

In all these trials the computational times lie between 6 and 8 minutes. This computational level should be compared with that of traditional MIP models as already reported in Section 3. Such models can only 
be implemented for very small applications as noted by O'Hara et al [15]. The present Whangapoua Forest case study is far too large to model as a traditional MIP formulation. Such a model would require explicit representation of all possible road harvest plans which in this case would be many millions. It is significant that the present model not only optimises large $\mathrm{r}$ problems than those mentioned in Section 3, but it also requires much less computational time.

\section{CONCLUSIONS}

The idea of developing an integrated model has been vindicated. The use of column generation in association with constraint branching has provided the technological power to achieve this end. The advantages of an integrated model that addresses both tactical and strategic issues are immediately apparent from the results of the case study presented above. When these are compared with the performance levels attained by other research workers addressing similar problems, as outlined in Section 3 , it is seen that significant advances have been made. High quality objective values have been attained. Also the solution time required is very short. Application size appears to present no problem, with the appropriate numerical trials indicating no undue escalation of either elapsed user time, or memory requirements, when the size of the model is increased.

The most significant line of associated research concerns the development of models for other applications with characteristics similar to those noted in Section 2.

\section{Acknowledgments}

This research is supported by the Forest Research Institute, Private bag 3020, Rotorua, New Zealand.

\section{References}

[1] D.K. Daust and J.D. Nelson (1993), Spatial Reduction Factors for Strata-Based Harvest Schedules, Forest Science, 39, pp. 152-165.

[2] M. Desrochers, J. Desrosiers and M. Solomon (1992), A New Optimisation Algorithm for the Vehicle Routing Problem with Time Windows, Operations Research, 40, pp. 342-354.

[3] D.S. Hochbaum and A. Pathria (1997), Forest Harvesting and Minimum Cuts: A New Aroach to Handling Spatial Constraints, Forest Science, 43, pp. 544-554.

[4] J. Hof and T. Baltic (1991), A Multilevel Analysis of Production Capabilities of the National Forest System, Operations Research, 
39 , pp. $543-552$.

[5] J. Hof (1993), Coactive Forest Management, Academic Press, San Diego.

[6] J. Hof, M. Bevers and J. Pickens (1996), Chance-Constrained Optimisation with Spatially Autocorrelated Forest Yields, Forest Science, 42 , pp. 118-123.

[7] H.M. Hoganson and J.G. Borges (1998), Using Dynamic Programming and Overlaing Subproblems to Address Adjacency in Large Harvest Scheduling Problems, Forest Science, 44, pp. 526-538.

[8] M. Kirby, W. Hager and P. Wong, (1986), Simultaneous Planning of Wildlife Management and Transportation Alternatives, TIMS Studies in the Management Sciences, 21, pp. 371-387.

[9] B.R. Manley, J.A. Threadgill and S.J. Wakelin (1991), Alication of FOLPI : A Linear Programming-based Forest Estate Model, Ministry of Forestry, FRI Bulletin 164, New Zealand.

[10] B.J. Meneghin, M.W. Kirby and J.G. Jones (1988), An algorithm for writing adjacency constraints efficiently in Linear Programming Models, in Proceedings of the 1988 symposium on Systems Analysis in Forest Resources, USDA For. Serv. Gen. Tech. Rep., pp. 46-53.

[11] A.J. McNaughton (1998), Long-term Scheduling of Harvesting with Adjacency and Trigger Constraints, Ph.D. thesis, Univ. of Auckland, Auckland, New Zealand.

[12] A. Murray and R. Church (1996), Analyzing Cliques for Imposing Adjacency Restrictions in Forest Models, Forest Science, 42, pp. 166-175.

[13] J. Nelson and J.D. Brodie, (1990), Comparison of a Random Search Algorithm and Mixed Integer Programming for Solving Area-based Forest Plans, Canadian Journal of Forestry Research, 20, pp. 934942.

[14] J. Nelson, J.D. Brodie and J. Sessions (1991), Integrating ShortTerm Area-Based Logging Plans with Long-Term Harvest Schedules, Forest Science, 37 (1), pp. 101-122.

[15] A.J. O'Hara, B.H. Faaland and B.B. Bare (1989), Spatially Constrained Timber Harvest Scheduling, Canadian Journal of Forestry Research, 19, pp. 715-724.

[16] D.M. Ryan (1992), Solution of Massive Generalised Set Partitioning Problems, Journal of the Operational Research Society, 43 (5), pp. 459-467. 
[17] J. Sessions and J.B. Sessions (1992), Tactical Harvest Planning Using SNAP 2.03, in Proceedings of Conference of International Union of Forestry Research, pp. 112-120.

[18] H.D. Sherali, W.P. Adams and P.J. Driscoll (1998), Exploiting Special Structures in Constructing a Heirachy of Relaxations for 0-1 Mixed Integer Problems, Operations Research, 46, pp. 396- 405.

[19] S. Snyder and C. ReVelle (1996), The Grid Packing Problem: Selecting a Harvesting Patter in an Area with Forbidden Regions, Forest Science, 42, pp. 27-34.

[20] S. Snyder and C. ReVelle (1997), Dynamic Selection of Harvests with Adjacency Restrictions: the SHARe Model, Forest Science 43, pp. 213-222.

[21] A. Weintraub and A. Cholaky (1991), A Hierarchical aroach to Forest Planning, Forest Science, 37, pp. 439-460.

[22] A. Weintraub, F. Barahona and R. Epstein (1994), A Column Generation Algorithm for Solving General Forest Planning Problems with Adjacency Constraints, Forest Science, 40 (1), pp. 142-161.

[23] A. Weintraub, G. Jones, M. Meacham, A. Magendzo, A. Magendzo and D. Malchuk (1995), Heuristic Procedures for Solving Mixed-Integer Harvest Scheduling-Transportation Planning Models, Canadian Journal of Forest Research, 25, pp. 1618-1626.

[24] A. Yoshimoto, J.D. Brodie and J. Sessions (1994), A New Heuristic to Solve Spatially Constrained Long-term Harvest Scheduling Problems, Forest Science, 40, pp. 365-396. 